\title{
El Presente Potencial y la Conciencia Histórica: Realidad Social, Sujeto y Proyecto. A la memoria de Hugo Zemelman Merino
}

\author{
Juan Pablo Paredes P. \\ Universidad Diego Portales, Santiago, Chile. \\ Email: paredesjp@gmail.com
}

\begin{abstract}
Resumen: El siguiente escrito ${ }^{1}$ tiene por finalidad rendir un pequeño homenaje académico a la figura y obra intelectual del epistemólogo chileno Hugo Zemelman Merino, recientemente fallecido. Para ello se expone la base de su principal contribución a la epistemología social latinoamericana, denominada epistemología del presente potencial y la consciencia histórica. En segundo momento revisamos los alcances sociohistóricos (a) y sociopolíticos (b) de la propuesta, en lo que lo acerca a un diálogo con las ciencias sociales más allá de los alcances epistémicos y metodológicos. En tercer lugar, a manera de cierre, realizamos una pequeña evaluación de lo expuesto sobre su obra, a la luz de un ángulo de lectura sociopolítico.
\end{abstract}

Palabras clave: Zemelman, realidad social, presente potencial, consciencia histórica, modo de concreción, sujetos sociales.

\section{Potential Present and Historical Consciousness: Social Reality, Subject and Project. In memory of Hugo Zemelman Merino}

\begin{abstract}
The following paper is intended to pay a small tribute to the academic and intellectual work of chilean epistemologist Hugo Zemelman Merino, who died recently. In so doing, the bases of his main contribution to Latin American social epistemology, called «epistemology of present potential and historical consciousness» is exposed. Secondly, we review the socio-historical (a) and sociopolitical (b ) extent of the proposal, as it approaches a dialogue with the social sciences beyond the epistemic and methodological approach. Thirdly, as a closure, we performed a small evaluation of his work, in the light of a reading from a socio-political angle.

Key words: Zemelman, social reality, present potential, historical consciousness, realization mode, social subjects.

\section{O Presente Potencial ea Consciência Historica: A Realidade Social , Sujeito e Projeto. Em memória de Hugo Merino Zemelman}

Resumo: O escrito a seguir destina-se a pagar uma pequena homenagem ao trabalho acadêmico e intelectual do epistemólogo chileno Zemelman Hugo 
Merino, que morreu recentemente . Para fazer isso está exposto baseado em sua principal contribuição para a epistemologia social latino-americana , chamada de «epistemologia dessa consciência potencial e histórico». Em segundo lugar, rever o momento sócio-histórico (a) e do âmbito político-social (b) da proposta, uma vez que se aproxima de um diálogo com as ciências sociais além do abordagem epistêmica e metodológica. Em terceiro lugar, como um fechamento, foi realizada uma pequena avaliação do acima, em sua obra, à luz da leitura do ângulo sóciopolítica.

Palavras-chave: Zemelman, realidade social, presente potencial, consciência histórica, modo de realização, sujeitos sociais

\section{Introducción}

Recientemente, en Octubre del 2013, fallece en México uno de los intelectuales críticos latinoamericanos más destacados de las últimas décadas: El epistemólogo chileno Hugo Zemelman Merino. Abogado y sociólogo de formación inicial, con una maestría en sociología de FLACSO, fue director de la Escuela de Sociología en la Universidad de Chile entre 1967 a 1970. Después fue un colaborador del Gobierno de Salvador Allende y debido al golpe militar del 1973, debe exiliarse en México. En este país es donde desarrolla su potente reflexión epistémico-categorial y metodológica, consolidando su trabajo académico y desplegando su vocación de intelectual crítico, que ya arrastraba desde Chile. En México es profesor titular de El Colegio de México (COLMEX), también colaboró con las instituciones más prestigiosas del país como la Universidad Nacional Autónoma de México (UNAM) y la Facultad Latinoamericana de Ciencias Sociales (FLACSOMéxico). Además fue profesor invitado en numerosas Universidades tanto en América Latina y España. Sus últimos años los pasó muy comprometido con la realidad latinoamericana, especialmente con países como Colombia, Argentina, Bolivia y por supuesto, su contradictorio Chile. La última etapa de su vida la dedicó a uno afianzar y potenciar uno de sus tantos proyectos intelectuales el Instituto de Pensamiento y Cultura en América Latina (IPECAL), del que fue cofundador y su primer director, que cuenta con sedes en México, Colombia y Chile. Sus publicaciones abarcan problemas agrarios, movimientos sociales, los regímenes militares y el autoritarismo, cultura política y el Estado, problemas educativos, la subjetividad, la historia y la política, desde un ángulo que daba prioridad a la entrada metodológica y epistemológica.

Como puede apreciarse, la obra de Zemelman es de una complejidad desbordante para sintetizarla en un breve escrito homenaje, abordando figuras como la emergencia, lo potencial, el presente, la memoria, la utopía, el sujeto, la realidad, la indeterminación o la articulación; considerando procedimientos metodológicos como la problematización, los círculos de reflexión, el análisis de coyuntura y los constructos no-parametrales; planteando lógicas de pensar como la apertura y la inclusión de dinámicas y procesos, lo epistémico y lo categorial versus lo teórico. Por tal razón, a 
manera de cumplir con el objetivo de homenajear la figura y el nombre del intelectual crítico Hugo Zemelman, presentaremos su obra a partir de lo epistémico, en base a sus dos pilares programáticos, que él mismo utilizo para denominar su propuesta epistemológica: el presente potencial y la consciencia histórica. A partir de estos dos pilares (primera parte del texto) desplegaremos ciertos contenidos que llamaremos sociohistóricos (segunda parte a del texto) y sociopolíticos (segunda parte b) implicados en su propuesta. Terminamos con una pequeña evaluación del valor de su obra desde lo político, así como los desafíos que nos lega (tercer apartado).

\section{Epistemología del Presente Potencial y la consciencia histórica}

La propuesta del presente potencial, esto es la aceptación de una ontología social anclada en el proceso de construcción constante e inacabado de la realidad social, que Zemelman llamará el movimiento de la realidad (1992), nos dice que tal movimiento es posible de ser aprehendido por los sujetos sociales. Tal capacidad inacabada de realidad no es un producto de una falla o vacío estructural que actuaría de horizonte de posibilidad de un excedente de sentido, como es en el pensamiento político-crítico actual, de base lacaniana, de Ernesto Laclau o Slavoj iek. Más bien en Zemelman se ve a la realidad como un proceso de no finitud o cierre, abierto a horizontes potenciales debido a la pluralidad de direcciones posibles en la construcción de la realidad, es decir, a la infinidad finita de caminos que puede seguir el movimiento de la realidad social. En otros términos lo inacabado de la realidad social es producto de los movimientos y de las múltiples direcciones de lo social, no por una falla estructural en ella sino por la imposibilidad del no-movimiento, por su exceso ${ }^{2}$. Así la idea de Zemelman es mucho más cercana a la idea de magma en Castoriadis, que a la falla estructural o falta en Lacan. Sin embargo esta lógica de lo abierto, no implica la inexistencia de operaciones de condensación de la realidad, que se traduce en un juego de problematización entre la determinación y la determinabilidad o definición ${ }^{3}$.

Llevado al plano del conocimiento pensar lo inacabado de la realidad significa intentar aprehender el movimiento siempre inconcluso de la realidad. Hugo Zemelman ha aceptado el desafío de aprehender el movimiento de la realidad, desde un pensamiento también móvil. Esto significa siguiendo a Luminato un cambio de ángulo: pasar desde la cuestión de la ontología social a la epistemológica, entendida esta como una lógica de razonamiento (Luminato, 1995: 30-31). Por ende el presente potencial liga desde el comienzo el exceso de realidad social dado por su constante movimiento, con la capacidad de aprehensión de las direcciones posibles por parte del conocimiento social. Así la epistemología del presente potencial relaciona en un nudo indisociable movimientos de la realidad social y posibilidades de conocimiento social. 
Zemelman propone la epistemología del presente potencial (1987: 23-32) como la forma lógica de conocer-aprehender el movimiento de la realidad social, donde la realidad social se entiende como una articulación de procesos heterogéneos en base a tres presupuestos:
a) el supuesto del movimiento
b) el supuesto de la articulación procesal
c) el supuesto de la direccionalidad.

Estos tres presupuestos permitirán elaborar una estrategia epistémica que no limite la potencialidad de la realidad social, pero a la vez que permita la expresión de las potencialidades del conocer, así Zemelman nos propone un ejercicio de imaginación epistemológica al relacionar el movimiento mismo de la realidad social en el movimiento del pensar y viceversa como procesos co-constituyentes ${ }^{4}$. El supuesto del movimiento es la base de lo inacabado de la realidad, en cuanto a sus propios procesos de constitución, al considerar en la forma del pensar los dinamismos estructurales y coyunturales propios de la realidad en su proceso de producción. De esta manera, se intenta superar los postulados estáticos, entregados por la observación empírica ingenua que confunde los parámetros de observación teóricametodológica de la realidad social con la realidad social misma (Zemelman, 1987). En términos simples se confunde el mapa con el territorio, borrando a la vez los alcances performativos de la propia actividad de conocer.

El supuesto de la articulación nos dice que los procesos constitutivos de la realidad social no pueden desvincularse unos de otros, más que por una necesidad analítica, y mediante un ejercicio de reconstrucción es posible proponer la emergencia de puntos de articulación. Estos puntos se condicen con la complejidad de la realidad social, entendida como un conjunto heterogéneo de procesos y planos vinculados entre sí (1987). Por su parte el presupuesto de la direccionalidad nos dice que los procesos sociales poseen múltiples direcciones posibles aunque algunas de ellas son más factibles que otras de ser actualizadas. Estas direcciones aunque contingentes no son al azar, pero tampoco movimientos teleológicos sino construcciones posibles dentro de los dinamismos propios de la realidad so$\mathrm{cial}^{5}$. Esto es que la realidad social permite la inclusión de la práctica de los sujetos sociales, así como de otros dinamismos constituyentes del propio movimiento como pueden ser algunas estructuras sociales. Sin embargo, el presupuesto de la direccionalidad es propio del movimiento de la realidad y no algo solo atribuible a los deseos de los sujetos. Con esto su pensamiento se escapa y supera del voluntarismo subjetivo, al formular la posibilidad de pensar sujetos con capacidad de politicidad en su accionar, pero siempre con el límite del movimiento entre lo dado-dándose.

El presupuesto de la direccionalidad se relaciona con el presupuesto de la articulación de procesos para conformar los movimientos de la realidad social, acá es donde aparecen ciertos componente performativos del conocer que Zemelman revisará en sus últimas obras vía la tarea del lenguaje (2007). Es la articulación de procesos la que dejará ver las posibles 
direcciones y orientaciones que asumirán los procesos sociales que conforman el movimiento de la realidad social. Sin embargo la tarea del conocer es observar las potencialidades de desarrollos posibles de la realidad y no solo la orientación actual de ella, por eso se habla del presente potencial (Zemelman 1987, 1992, 1994)

El planteamiento basal de la epistemología del presente potencial, es la de abrir el razonamiento desde un ejercicio que rompe los parámetros autoimpuestos por formas cerradas de conocer, por ejemplo la practica científica positivista, hacia una forma de conocimiento-aprehensión del movimiento de la realidad social a través de un procedimiento problematizador de la realidad, basado en una lógica de inclusión permanente de procesos, niveles y conceptos. La finalidad es la construcción de una matriz categorial que permita la reconstrucción articulada de la realidad social sin simplificar su complejidad inherente, a través de una relación de conocimiento del mismo tipo. Un planteamiento tal requiere el posicionamiento del sujeto, un ángulo de lectura, que desde la propuesta zemelmaniana, está anclado en la subjetividad y los sujetos sociales.

Es desde la tarea del conocer que un sujeto concreto y situado, no trascendente ni formal, elaborará un ángulo de lectura/apropiación de la realidad a fin de conocerla. La tarea de conocer en Zemelman está ligada a la práctica y acción de los sujetos, así como a las materialidades y significaciones dadas por el lenguaje. La consciencia histórica refiere a la capacidad de los sujetos de colocarse frente a la realidad, bajo un criterio de construcción histórica, de tal forma que la realidad social deja de ser simplemente una externalidad para los sujetos para entenderla como una constelación de ámbitos y de múltiples sentidos posibles (2002).

Es vía la noción de consciencia histórica que Zemelman plantea el doble vínculo entre sujeto y realidad histórica, como co-constituyentes, al no limitar la capacidad de conocer de los sujetos a las determinaciones posibles por la realidad histórica. Los sujetos son limites dados, pero a la vez son potencialidades ya que cuentan con la capacidad de trascender los límites conceptuales con los que se piensa en un determinado momento, expresada en la potencialidad de resignificar y dar nuevos contenidos de los sujetos (2002). Esto es posible de observar tanto en la tarea de investigar la realidad social, como en el mundo de la vida cotidiana. Desde el plano de la cotidianeidad se observa la capacidad de los sujetos de transformar lo indeterminado o lo naturalizado en otras modalidades de contenidos y sentidos. Para esto Zemelman rescata la noción de asombro en el conocer (2002), donde el asombro configura la potencialidad de la apertura propia de la realidad social y del conocimiento sobre ella, dándole una forma que puede parecer ingenua, pero que es productiva en tanto produce nuevas definiciones.

En el fondo la consciencia histórica se ancla en las capacidades y potencialidades de los sujetos de romper y desarmar las estructuras que naturalizan el conocer, de paso que naturalizan a la misma realidad social, 
conjunto a las posibilidades de configurar un pensamiento desparametrizador de aquellos parámetros que actuaban como bloqueos, permitiéndose potenciar las diferentes direcciones de los movimientos de la realidad, bajo la tensión posible entre un conocer desparametrizador y la realidad social naturalizada.

No obstante lo anterior, no debe perderse de vista que la formulación de Zemelman es profundamente relacional, en tanto la realidad social y sus direcciones condensadas actuarán de cierta forma sobre la capacidades de conocer de los sujetos. Pero estos están en condiciones de relacionarse con ella de manera distinta, dada por las potencialidades de su conocer (lenguaje y práctica), de una manera tensional que explora los diferentes movimientos de la realidad social, así como la experimentación de otras direcciones, donde realidad social y sujetos son nodos indisociables. Este punto de vista tensional requiere profundizar en las formulaciones sociohistóricas o de historicidad y en las sociopolíticas presentes en la propuesta del presente potencial y la consciencia histórica, para observar las implicancias sociológicas y políticas de la propuesta.

\section{Dinámica de la Historicidad: Modos de concreción y subjetividad en la realidad social}

La propuesta de Zemelman, en contraposición a ciertas orientaciones que privilegian lo fragmentario y la dispersión de los procesos sociales en la actualidad, intenta reposicionar la pertinencia y relevancia de la categoría de totalidad en el análisis social. Las funciones de la totalidad, entendida como exigencia de un razonamiento complejo acorde a la realidad social, implican la relación entre las prácticas de los sujetos sociales y las formas de estructurarse de la realidad social en un espacio-tiempo determinado $(1989,1992,1995)$. La totalidad es la forma de organización de un razonamiento en base a la inclusividad de categorías, niveles y procesos, para reconocer la articulación en que estos encuentran su específica significación (1992: 50). Concebida la totalidad como una forma de organizar el razonamiento categorial, en base a una exigencia de inclusión, implica una construcción que aprehende lo real en tanto constituyéndose en un movimiento dado-dándose ${ }^{6}$ (1992: 53-54).

Una exigencia de totalización requiere pensar las relaciones que se establecen entre los planos objetivos y subjetivos de la realidad social, en cuanto a que ambos son constituyentes de la historicidad del movimiento de la realidad social. La historicidad alude tanto al momento presente, como a la potencialidad contenida en la realidad social por el movimiento de sus procesos y elementos constituyentes. La idea de historicidad refiere inmediatamente a la indeterminación de lo social por su propio movimiento y exige la relacionalidad de sus elementos para considerar la realidad como dada-dándose. En tal sentido, Zemelman nos propone considerar a la realidad como histórica, ya que es un campo abierto de acciones alternativas 
capaces de crear realidades. En otras palabras refiere a las fuerzas sociales que construyen realidades en tanto direcciones posibles $(1992,1996)$.

La inclusión de fuerzas sociales en la construcción de las realidades sociohistóricas señala la implicación de subjetividades y sus prácticas en la estructuración de lo social. Por ende, tanto estructura como acción son elementos constitutivos de la historicidad de la realidad social. Sin embargo la noción de estructura debe ser replanteada para adecuarse a las necesidades del movimiento de la realidad. Zemelman nos propone sustituir el concepto de estructura por el de modo de concreción, entendido como una determinada conjugación de niveles y momentos, en un ritmo articulado que considera la relación dinámica entre estos, es decir un proceso de condensación de la relación articulada en una determinada dinámica temporo-espacial (Zemelman 1992: 93-94). De esta manera, un modo de concreción no es nunca algo cerrado ni acabado, sino una construcción que permite la inclusión de nuevos niveles y procesos en su dinámica, por ello es un modo de concreción abierto a sus modificaciones.

Pensada así la estructura de lo social, como el modo de concreción de un dinamismo específico, autoriza a considerar a la acción de los sujetos sociales constitutiva de un modo de concreción particular, más específicamente no es solo la acción de los sujetos sino que la subjetividad misma es constituyente de un modo de concreción determinado, con lo que la oposición clásica entre acción y estructura o subjetivo y objetivo pierde pertinencia ${ }^{7}$. Porque, de acuerdo a como se ha presentado la reflexión, ambos son pilares constitutivos en la historicidad de la realidad social, así como de su conocimiento.

La cuestión de la subjetividad pasa a ser fundamental, ya que no solo es co-constituyente del movimiento de la realidad sino que también es intervenida por el mismo movimiento de la realidad, produciendo una mutua relación de producente-producido entre realidad y sujetos, como de sujetos y realidad ${ }^{8}$. Para Zemelman la relevancia de la subjetividad y de los sujetos es que son condensadores de historicidad, ya que en ellos confluyen y se reelaboran los diferentes procesos de la dinámica social. Al ser condensadores del movimiento de la realidad social participan de la dinámica dado-dándose, presentando diferentes alternativas de activación y potenciación de ella, mediante la apropiación de la historicidad social. Pero una subjetividad constituyente-constituida no puede reducirse a un mero efecto o a una interioridad de la estructura, sino que requiere una conceptualización más amplia. Zemelman nos dice respecto a la subjetividad:

dado su carácter estructurado-estructurante no puede entenderse [la subjetividad] como un campo definido en términos de sus manifestaciones, ya sean conductuales, de expectativas o perceptivas, sino de modo más profundo, desde su misma dinámica constitutiva y constituyente: lo que nos remite a campos de realidad más profundos. (Zemelman, 1996: 104) 
Con lo anterior queremos especificar que concordamos con Zemelman en que la subjetividad es un elemento que permite la emergencia de campos problemáticos más extensos y profundos. La subjetividad actúa como punto articulador de diversos planos y niveles tanto estructurales, como culturales y psicológicos. Pero la subjetividad en Zemelman es parte del problema de la construcción de la realidad-capacidad de conocerla y no la respuesta exclusiva, en tanto promesa emancipatoria. En simple es una potencialidad.

Lo anterior facilita tratar a las subjetividades como hechas y por hacer, es decir como efectos de determinados dinamismos de la realidad, pero simultáneamente como emergentes, es decir portadoras de lo inédito y lo potenciable de la realidad. Esto dependerá de las articulaciones propias de las subjetividades en el proceso de conformación la realidad social y de los sujetos sociales.

\section{Dinámicas de Historicidad: Nucleamientos de lo colectivo en la constitución de sujetos sociales}

Tal como se planteó anteriormente, la subjetividad es para Zemelman necesariamente colectiva, implica lo individual pero es social al ser constitutiva del movimiento de la historicidad de la realidad. Por ende se debe tematizar las formas en que las subjetividades colectivas se constituyen como sujetos sociales, en un proceso donde confluyen lo material, como lo inmaterial, las prácticas, las expresiones de la conciencia, los diferentes lenguajes, los diferentes planos sociales, etc. Tanto en las instancias en que las subjetividades son actuadas, como en aquellas en las que actúan y que son reactuadas por su propia acción, lo que implica necesariamente un proceso de ampliación y complejización de la realidad.

El proceso desde el que las subjetividades colectivas se transforman en sujetos sociales implica para Zemelman, la articulación de varios niveles de la realidad, así como de diferentes temporalidades y escalas espaciales, donde confluyen elementos psicológicos (deseo y necesidad), socioculturales (memoria, práctica, proyecto, utopía) y estructurales-económicos (prácticas habituales). Zemelman denomina a estos procesos nucleamientos de lo colectivo, que permitirán ciertas cristalizaciones culturales en la conformación de sujetos pero nunca su inmutabilidad (1996).

Los nucleamientos colectivos son convergencias de procesos y espacios de constitución de fuerzas colectivas que pueden impulsar la construcción de la realidad social en diferentes direcciones (1996: 21-22). Estos procesos-espacios son definidos, por Zemelman, en torno a tres ejes:

- La necesidad entendida, no como es pensada en términos tradicionales de carencia, sino cómo como expresión constitutiva de la realidad social en su dimensión temporal y espacial. Es decir necesidad 
refiere a un dinamismo propio de lo social: la necesaria relación entre memoria y utopía (pasado y futuro) así como entre lo microsocial y macrosocial o lo objetivo-subjetivo.

- La relación entre memoria y utopía, así como entre planos, permite la emergencia de experiencias, que se refieren al presente entendido como lo objetivo de la situación, la transformación de lo deseable/ potencial en posible. Es el espacio de despliegue de prácticas colectivas con anclaje en la memoria y experiencia, pero abiertas al futuro. - La experiencia en cuanto se refiere a determinados modos de concreción posibilita la construcción de proyectos entendidos como las modalidades en las que las múltiples posibilidades de desenvolvimiento de la realidad se expresan como opciones posibles/viables.

La vinculación entre necesidad, experiencia y proyecto conforman la base para los nucleamientos de lo colectivo. De esta forma, los diferentes niveles que participan de la construcción de la realidad social se relacionan: lo individual, lo grupal, las voluntades colectivas (1996). Estos niveles se articulan en función tanto de las dos dimensiones de las subjetividades: como producto así como productor.

De esta forma Zemelman propone un esquema de análisis de las modalidades de los nucleamientos colectivos, que van de lo individual en planos grupales hasta la conformación de voluntades colectivas capaces de implementar proyectos con la pretensión de direccionar el movimiento de la realidad social. Un ejemplo de lo que señalamos es la noción de sujetos comunitarios que Zemelman presenta en uno de sus escritos, pero que antes ya señalaba en diferentes modalidades de nuclear lo colectivo $(1987,1996)$.

En síntesis de acuerdo a la propuesta de Zemelman, considerar la realidad desde la subjetividad constituyente en consideración de las diferentes modalidades en que se nuclean colectivamente las subjetividades, para la conformación de sujetos sociales, posibilita ingresar la dimensión conflictual en la historicidad de la realidad social, en tanto la construcción de múltiples posibilidades implica la existencia de más de un sujeto social y sus prácticas. Se requiere de un análisis de los procesos que sedimentan, compiten y fracturan los parámetros existentes en el enfrentamiento de diferentes sujetos y sus acciones por los modos de concretar las potencialidades de la realidad ${ }^{9}$. Esto es pasar de la sociohistórico a la política.

\section{La dimensión sociopolítica: Voluntades Colectivas y Proyectos en la Historicidad de lo social.}

Una concepción dinámica de los procesos históricos es incompleta sino introduce la dimensión de poder y conflicto en la construcción de la historicidad. Si se considera que la construcción sociohistórica, en términos de orden social (sedimentación del movimiento), es resultado de la lucha entre diferentes proyectos impulsados por diversos sujetos sociales, en tanto estos encarnan concepciones antagónicas sobre el futuro (1989: 13), no es posible marginar las relaciones de poder en la construcción de la 
realidad. Esto implica avanzar desde la subjetividad emergente-constituida hacia la implicación y el papel que los sujetos sociales- a través de sus prácticas- juegan en los procesos de construcción de la realidad.

Al considerar la conjunción entre sujetos sociales y prácticas de apertura a lo potencial de la realidad, el movimiento de ella y sus modos de objetivación, son el resultado de una sedimentación conflictual. Esto implica necesariamente incorporar en el centro de los procesos de historicidad de la realidad social a la dimensión política, ya que la acción de los sujetos sociales no es nunca de un solo tipo: puede ser un tipo de acción que beneficie la sedimentación o puede ser un tipo de acción que formule nuevos dinamismos y articulaciones, orientadas a lo potenciable/posible/deseable ${ }^{10}$. La dimensión de lo político es constitutiva del movimiento de la realidad ya que permite romper las naturalizaciones propias de la sedimentación de la historicidad del presente. Pero no significa necesariamente la reducción del movimiento de la realidad a lo político, sino que es uno de sus dinamismos más significativos.

Lo político será para Zemelman la articulación dinámica entre sujetos, prácticas sociales y proyectos impulsados por estos mismos, cuyo contenido es la lucha por la direccionalidad de la realidad en el marco de opciones viables (1989: 13). De tal forma la inclusión de los dinamismos políticos en la construcción de la realidad social implica necesariamente las acciones impulsadas por voluntades colectivas en base a sus proyectos. Lo central para un planteamiento que se centra en la dimensión política de la construcción de la realidad, es explotar la objetividad potencial contenida en ella, en base a la acción de sujetos sociales (1989:14)

Considerar esta dimensión conflictual significa privilegiar un ángulo de lectura desde el cual los sujetos sociales imprimen a sus prácticas la politicidad necesaria para fracturar y romper los parámetros establecidas por la sedimentación temporo-espacial de la realidad como orden social. La dimensión política-conflictual, al decir de Zemelman, considera la toma de conciencia por parte de los sujetos sociales de la historicidad de la realidad social, en tanto construcción de proyectos colectivos que se apropian en lo posible del movimiento de la realidad (1989:18). Esto es considerar a los sujetos sociales en forma de voluntades colectivas. Para Zemelman los sujetos sociales vistos como voluntades colectivas implican un ángulo de lectura que privilegia lo producente sobre lo producido, es decir pensar en términos de potencialidades del movimiento de la realidad hacia el futuro o en otras palabras: la capacidad de actuar colectivamente.

Las voluntades colectivas son para Zemelman las formas que asumen los sujetos sociales en la tarea por apropiarse del futuro ${ }^{11}$, en tanto capacidad de reactuar sobre lo dado, con la finalidad de hacer emerger lo potenciable sobre lo real-objetivo-dado (1989: 20-40). Pero las voluntades colectivas no son solo imaginación utopística o puro deseo, son también base de experiencia histórica, ya que como todo sujeto social son condensaciones de la historicidad de la realidad social. En otros términos 
las voluntades colectivas no serían las multitudes de Negri, ya que implican formas determinadas de articulación colectiva en base a los diferentes procesos de nucleamientos colectivos que experimenten. En este punto se diferencian de la propuesta de Laclau y la articulación en base a significantes vacíos $^{12}$ que permitirían articulaciones más formales sin tanto peso en las bases históricas, en otros términos no es posible cualquier articulación entre sujetos. Así las voluntades colectivas son una expresión de los sujetos sociales que en su acción imprimen la dimensión del poder en el intento de dinamizar el movimiento de la realidad en otras direcciones, pero siempre con un anclaje en su propia historia. Lo que refiere a densidad histórica en su formación con lo que no podrían ser meras posiciones en marcos discursivos.

La experiencia histórica le permite a las voluntades colectivas experimentar los diferentes caminos de apropiación de los movimientos de la realidad con un anclaje en sus propios derroteros, aunque siempre abiertos a la novedad de la historicidad, esto a través de los reconocimientos de los horizontes históricos que, en palabras de Zemelman, resultan de la compleja y variable articulación de procesos sociales en los cuales se producen las alternativas posibles (1989: 33)

La forma de intervenir en estas articulaciones es mediante el ejercicio del poder de las voluntades colectivas, entendido este poder como actuación-decisión sobre lo real a la vez como potencialidad expresado en proyectos colectivos de apropiación del movimiento de la realidad. Para Zemelman esto significa la capacidad de transformar la utopía (dimensión de futuro) en historicidad presente. Los proyectos son las formas de plantear los procesos de apropiación del movimiento de la realidad en términos de direccionalidad dentro de horizontes históricos. Sin embargo un proyecto no es la definición de una meta o tarea específica por cumplir. Al contrario, es la aceptación del desafío de aprehender lo abierto del movimiento de la realidad social, lo que significa entender que el mismo proyecto es a su vez movimiento: abierto e inacabado. Esto supone conciencia histórica, como reconocimiento del movimiento en sus posibilidades de apropiación en tanto vislumbrar los límites/potencialidades de la situación. En cambio la meta es justamente lo contrario: la creencia en la cancelación del movimiento de la realidad, con lo cual el concepto de utopía pierde su riqueza política para transformarse en sinónimo de totalitarismo.

Los proyectos de apropiación de la realidad por los sujetos sociales, suponen la inclusión de la utopía. Está en el presente potencial no es la existencia del futuro como ley, sino es la potencialidad histórica de actualización de direcciones de la realidad en base al principio de apertura. En la relación utopía (lo abierto de la realidad como posibilidades) -proyecto (como viabilidad de la apropiación del movimiento de la realidad) se expresan las voluntades colectivas en tanto forma de rescatar la dimensión política de la historicidad contra la reificación de la realidad social (1989: 50-53). Visto desde tal óptica, la cuestión política de la historicidad impli- 
caría tanto ruptura del no-movimiento de lo real, como sedimentación de un estado, a la vez como continuidad en el propio movimiento, en cuanto nuevo dinamismo. Para esto nos propone pensar a los sujetos sociales como voluntades colectivas capaces de implementar proyectos de apropiación del movimiento de la realidad que en base a la utopía (la posibilidad siempre abierta de la realidad) van materializando posibilidades de nuevas emergencias en la realidad.

Las posibilidades significan para los sujetos sociales el ejercicio de la activación de las potencialidades de la realidad y el ejercicio de la elección en sus prácticas en función de nuevas emergencias. De manera tal que lo político conlleva una lógica de la decisión por parte de los sujetos, de modo que lo político significa la transformación de ciertas posibilidades de futuro en formas de actividad práctica en el presente. Por lo mismo una lógica de la decisión es constitutiva de la realidad en movimiento como forma de vincular practica-proyecto (1989: 87). Es a través de la decisión que se interviene en el ritmo-movimiento de la realidad, desde la óptica política.

\section{Presente potencial, consciencia histórica, historicidad y lo político: una evaluación crítica.}

Las decisiones de los sujetos vinculan la acción en tanto proyecto como apropiación del movimiento de la realidad, pero nada asegura que esta acción-proyecto sea una apropiación emancipatoria. Tal como se expuso antes es una apropiación sin garantías, contingente. La pregunta sobre si las potencialidades de la realidad aseguran proyectos orientados a la apropiación del movimiento de la realidad en un sentido emancipatorio, es lo que queda sin formularse de manera directa por la propuesta del presente potencial, que privilegia el ángulo epistémico para formularse por sobre el político-normativo.

Este es el déficit directamente político, con secuelas ético-normativas, que Zemelman no desarrolla en su propuesta epistemológica. La cuestión que deja a medio camino es: ¿cómo se produce la conformación de voluntades colectivas emancipatorias? o en otros términos, qué asegura que el proyecto de apropiación de los sujetos sociales se plantee la relación entre acción y sentido emancipatorio ${ }^{13}$. Si seguimos los planteamientos de Zemelman, tal como lo hemos hecho hasta ahora, un proyecto encarna la conciencia de construcción de futuro y las prácticas requeridas para su logro, pero esto no asegura en nada que el proyecto sea emancipatorio o que sus prácticas lo sean. La brecha que se establece es entre potencialidad de la realidad- trabajada de manera excelente en la propuesta del presente potencial- y proyecto de orientación emancipatoria en el marco de la historicidad ${ }^{14}$. Una pregunta que se puede inmediatamente formular es: ¿la voluntad colectiva de tipo emancipatorio asegura en sí misma acciones de este mismo tipo? 
Una consecuencia de no desarrollar esta brecha que nosotros establecemos entre la cuestión de la decisión planteada en De la Historia a la Política y las prácticas emancipatorias es que no existe una problematización de la relación entre decisión y acción emancipatoria en los sujetos sociales. Podemos encontrar dos problemas en torno a la acción de los sujetos sociales que deben formularse, desde la óptica del presente potencial y la lógica de proyectos: a) la cuestión del sentido de la acción de los sujetos en tanto proyecto; b) la cuestión de la acción del sentido en el movimiento mismo de la acción. El primer problema se refiera al sentido previo que establece una decisión en cuanto a la acción misma, el segundo se refiere al movimiento mismo del sentido en las diversas acciones una vez que en ingresan en determinados ritmos y dinámicas de la historicidad. La cuestión es la relación que existe entre el sentido inicial de la acción y los sentidos en el proceso mismo de despliegue de la acción, en tanto participe de la potencialidad.

Por otro lado, el tema de la acción y sus sentidos, puede delimitarse en cuanto a posibilidad políticas al referirse a relaciones antagónicas. Sin embargo nuevamente esto no está lo suficientemente trabajado en Zemelman, aunque reconoce lo propio de la política como un campo de lucha de proyectos impulsados por diferentes sujetos sociales. La cuestión es que se da por supuesto el antagonismo y no se relaciona la acción y sus sentidos en la propia emergencia del antagonismo. Esto nos dice que perfectamente una apropiación del movimiento de la realidad pueda ser conservadora o regresiva. Es la dimensión de antagonismo, como conformación de contenidos antagónicos (conservadores o emancipatorios), la que no está trazada con la suficiente fuerza y claridad en Zemelman. En otras palabras se pueden producir rupturas y cambios de dirección en la construcción de la realidad pero nada asegura que estos sean orientados a la liberación. Entonces, el tema es la orientación de la voluntad colectiva hacia proyectos emancipatorios en la construcción de la historicidad, que signifiquen el cuestionamiento de la dominación y opresión, pero que se formulen en clave emancipatoria y subalterna. Sin embargo, por las dos críticas formuladas observamos en Zemelman una falta de reflexión sobre el vínculo entre acción emancipatoria y proyecto en función de constituir voluntades colectivas contrahegemónicas.

Ambas deficiencias convergen en lo que podríamos establecer como el déficit ético-normativo en la formulación política del presente potencial y la consciencia histórica. En Zemelman se observa una desvinculación de lo normativo, en tanto prefiguración de la dirección de la realidad, de los proyectos de futuro dentro de determinados marcos históricos. La pregunta que queda pendiente, y que autoriza la lectura realizada, a la obra de Zemelman es: cuáles son los contenidos mínimos de un proyecto emancipatorio que se formula en el marco de la historicidad de lo social, donde no todo puede advenir cualquier cosa. O en otro registro, ¿cuán política es la consciencia histórica de los sujetos sociales?

Las anteriores preguntas permiten formular otras, como por ejem- 
plo: ¿Cuáles serían los alcances éticos de los proyectos desde los sujetos sociales? y ¿cuáles los del investigador? De estas mismas preguntas, en tanto nos interesa la formulación de una matriz epistémico-metodológica para la investigación social que no sea del todo formalista, se puede desprender la pregunta por la implicancia del investigador y de su práctica en la implementación de los proyectos de carácter emancipador y en clave subalterna ${ }^{15}$.

Zemelman nos permite acercarnos a responder a las preguntas en sus últimas obras, en el sentido de colocar una posibilidad de respuesta situada en una forma más que en un contenido particular y definido. A partir de Necesidad de consciencia (2002), Zemelman vuelve a retomar la idea de los alcances éticos del conocer, colocando también los problemas de construcción de realidad y conocimiento en el orden del lenguaje (2007), en las tareas de los sujetos sociales (epistémico-políticos), en tanto condensadores de historicidad a la vez que catalizadores de cambios, en la construcción de la realidad bajo la óptica del tiempo y el espacio (2011b).

No obstante su muerte nos priva del desarrollo de tales respuestas. Pero, siendo profundamente zemelmanianos, tales ausencias de respuesta no son obstáculo, sino un desafío. Un desafío para el trabajo de conocer, para aprehender la realidad social desde los sujetos sociales y políticos. A la vez, Zemelman no nos deja en la intemperie de la cruda realidad naturalizada, nos hereda toda una rica y voluminosa obra que aporta en el trabajo de desnaturalizar la realidad y enfrentarse a la tarea del conocer, pero a la vez nos hereda una actitud, su voluntad y un compromiso crítico. Las bases están puestas, ahora es nuestra tarea mantener su legado. 


\section{Notas}

${ }^{1}$ Hugo Zemelman fue integrante del Comité Editorial de la Revista Polis desde sus inicios hasta su reciente deceso.

${ }^{2}$ En estos términos entendemos que la cuestión se refiere siempre a un excedente de realidad en ambas posiciones, pero en Zemelman es por el movimiento mismo, más que a un vacío o falta en ella. Aunque Slavoj Zizek presenta el vacío lacaniano en términos diferentes, en algunos aspectos, a los de Laclau. Pero este punto es una cuestión a trabajar a futuro, como programa de investigación sobre la ontología social y no se desarrolla en este escrito.

${ }^{3}$ Esto acerca a Zemelman a un posible diálogo no solo con Castoriadis, también a S. Hall que problematiza la relación de determinación y aboga por un pensamiento sin garantías.

${ }^{4}$ Esto es lo que podría acercar un diálogo entre el pensamiento de Zemelman y el de teóricos de la complejidad como E. Morin, J. Ibáñez o H. Maturana pero que en ningún caso se confunde con ellos.

${ }^{5}$ Esta direccionalidad posible es lo que Zemelman entenderá como lo objetivamente posible, es decir existe en la realidad un desenvolvimiento tendencial- potencial contenido dentro del propio movimiento de la realidad que no permite completamente al azar, pero tampoco la determinación total de los procesos. Lo objetivamente posible es aquello que permite ver vinculación de planos estructurales y simbólicos-subjetivos. Acá se juega la relación entre determinación y determinabilidad que señalamos más adelante.

${ }^{6}$ Esto podría permitir la recuperación de la idea de totalización-destotalización-retotalización propuesta por Sartre para la dinámica social. Ver Gruner 2002.

${ }^{7}$ En este sentido la propuesta de Zemelman entrega una alternativa para pensar esta relación diferente a la que entrega Bourdieu, Giddens, Beck o Habermas en sus respectivas formulaciones teóricas. Consideramos que un dialogo crítico con estas elaboraciones es muy necesario, para así acercar o alejar las diferentes posiciones. Nuestra intuición es que la alternativa zemelmaniana permite la emergencia de subjetividades rebeldes, así como las formula Sousa Santos (2006), en contraposición de las alternativas eurocéntricas que optan por una formulación más contenida o estructural de la subjetividad.

${ }^{8}$ En este sentido es que de la Garza Toledo, desarrollando el pensamiento de Zemelman, en su propia estructura teórica ha propuesto el concepto de configuración subjetiva.

${ }^{9}$ En la propuesta de Zemelman se observa la relación entre la vida cotidiana, los modos de estructuración sistémico y las dimensiones posibles del movimiento de la realidad, como lo aún no devenido, en lo que se refiere a la implicación de la utopía en la construcción de la realidad, en base al ejercicio de voluntades colectivas. Trabajamos esto en el siguiente apartado bajo el ángulo de lo político.

${ }^{10}$ Es necesario aclarar que pueden darse gradientes entre los polos que conforman la relación, entre el conformismo de la acción (rutinización de la naturalidad de lo social) o bien entre lo rebelde de la acción. La cuestión es la relación entre los sentidos y los momentos de la acción. Entre los muchos que han planteado el asunto ver de Ípola, 2001.

${ }^{11}$ Es importante acá recalcar que voluntades colectivas refieren a formas o construcciones 
categoriales no cerradas (no son conceptos), más que a contenidos empíricos. El tema es que voluntades colectivas, así como los proyectos, permiten construir un ángulo de lectura que pueda hacerse cargo de la dimensión política en la construcción de la realidad social a través de su problematización.

${ }^{12}$ Ver críticas de Stuart Hall a Laclau en Hall (1996)

${ }^{13}$ Este es un tema central en la actualidad cuando vemos la hegemonía del conformismo y la complacencia en cuanto a la realidad social. Como lo expone Lechner para inicios del 2000- hoy el desafío es contribuir a la desnaturalización de social (2002). Por lo mismo nuestra preocupación por los dinamismos y potencialidades de los sujetos políticos en la formulación de proyectos emancipatorios.

${ }^{14}$ De cierta forma Retamozo, en una propuesta mucho más avanzada que la nuestra, intenta enfrentar el mismo desafío al proponer un abordaje de los sujetos colectivos y los movimientos sociales que considera los sentidos de la acción en el marco de proyectos subalternos. Ver Retamozzo 2006.

${ }^{15}$ Con estas preguntas no queremos confundir a los lectores, el planteamiento de Zemelman es profundamente ético-político, al entender la realidad como un campo de posibilidades abiertas a su actualización y el uso del conocimiento para apropiarse del futuro son una forma de politización del quehacer investigativo-intelectual, sin embargo tiene un sesgo formalista. Nuestra intención es profundizar en una línea que consideramos no fue lo suficientemente trabajada en la obra de Zemelman, aunque si es enunciada desde el inicio de sus trabajos y la desarrolló en libros como De la Historia a la Política o en Problemas antropológicos y utópicos del conocimiento y la recupera, en parte, desde El Ángel de la Historia, pero ha sido subordinada a la reflexión epistémica-metodológica e incluso a la psicológica-cultural. Esta línea había sido retomada en sus últimos trabajos como horizontes de la razón III (2011b) y en el manuscrito aún inédito Cultura y poder retoma directamente los temas acá formulados, sin embargo su muerte nos deja la ventana abierta a tales preguntas. Por otra parte, Susana Luminato ha explorado las secuelas ético-normativas de la propuesta zemelmaniana. Ver Luminato 1994; 1995. 


\section{Bibliografía}

Butler, Judith; Laclau, Ernesto y Zizek, Slavoj (2003), Contingencia, Hegemonía y Universalidad. Diálogos contemporáneos en la izquierda. FCE. Buenos Aires, Argentina.

Castoriadis, Cornelius (1990), El mundo fragmentado. Altamira. Buenos Aires, Argentina.

Ídem (2006), Figuras de lo pensable. FCE. Buenos Aires, Argentina.

De Ípola, Emilio (2001), Metáforas de la política. Homo Sapiens. Rosario, Argentina.

De la Garza Toledo, Enrique (1992), Crisis y Sujetos Sociales en México. UNAM- Porrúa. México D. F, México.

Ídem (2001), “La epistemología crítica y el concepto de configuración”. Revista Mexicana de Sociología. 1/2001. UAM, pp. 109-127.

Dussel, Enrique (2006), 20 tesis de política. Siglo XXI. México D. F. México.

Escobar, Arturo (2005), Más allá del tercer mundo. Globalización y Diferencia. ICANH. Bogota, Colombia.

Gruner, Eduardo (2002), El fin de las pequeñas historias. De los estudios culturales al retorno (imposible) de lo trágico. Paidos. Buenos Aires, Argentina

Hall, Stuart y Grossberg, Lawrence (1996), "On the postmodernism and articulation. An interviews with Stuart Hall”. En Morley David, and Chen Kuan- Hising, Stuart Hall. Critical Dialogues in Cultural Studies. Routledge, Londres, Inglaterra.

Hall Stuart (1996), “The problem with ideologies. The Marxism with guarantees”. En Morley David, and Chen Kuan- Hising, Stuart Hall. Critical Dialogues in Cultural Studies. Routledge, Londres, Inglaterra.

Ibáñez, Jesús (1990), El retorno del sujeto. Amerindia. Santiago, Chile.

Laclau. Ernesto y Mouffe, Chantal (2001), Hegemonía y Estrategia socialista. Hacia una radicalización de la democracia. FCE. Buenos Aires, Argentina.

Laclau, Ernesto (2005), La razón populista. FCE. Buenos Aires, Argentina.

Lander, Edgardo [coord.] (2000), La colonialidad del saber: eurocentrismo 
y ciencias sociales. Perspectivas latinoamericanas. Faces/UCV. Caracas, Venezuela.

Lechner, Norbert (2002), Las Sombras del Mañana. La dimensión subjetiva de la política. LOM. Santiago, Chile.

Luminato, Susana (1994), “La función epistemológica de las utopías en la construcción del conocimiento social”. En Zemelman, Hugo [Coord] (1994), Círculos de reflexión latinoamericana en ciencias sociales. Cuestiones de teoría y método. Suplementos Anthropos 45. Anthropos. Barcelona, España.

Ídem (1995), "La función de los valores en el pensamiento filosófico latinoamericano”. En Zemelman, Hugo [coord] (1995), Determinismos y alternativas en las ciencias sociales de América Latina. CRIM-UNAM/Nueva Sociedad. Caracas, Venezuela

Mouffe, Chantal (2007), En torno a lo político. FCE. Buenos Aires, Argentina.

Negri, Antonio (2008), La fábrica de porcelana. Paidos. Barcelona, España.

Ranciére, Jacques (1996), El desacuerdo. Política y Filosofía. Nuevo Visión. Buenos Aires, Argentina.

Ídem (2006), Política, policía, democracia. LOM. Santiago, Chile.

Retamozo, Martin (2006), "Esbozos para una epistemología de los sujetos y movimientos sociales”. En Cinta de Moebio, 26. Revista Electrónica de Epistemología y Metodología de las ciencias sociales. Universidad de Chile. URL: http://www.facso.uchile.cl/publicaciones/moebio/26/retamozo.htm

Sousa Santos, Boaventura de (2005), El milenio huérfano. Ensayos para una nueva cultura política. Trotta. Madrid, España

Ídem (2006), Renovar la teoría crítica y reinventar la emancipación social. Clacso. Buenos Aires, Argentina.

Zemelman, Hugo (1987), Conocimientos y sujetos sociales. Contribución al estudio del presente. El colegio de México. México D.F, México.

Ídem (1989), De la historia a la política. La experiencia de América Latina. Siglo XXI. México D.F, México.

Ídem (1992), Los horizontes de la razón Vol. I y II. Antrophos. Barcelona, España.

Ídem [Coord] (1994), Círculos de reflexión latinoamericana en ciencias 
sociales. Cuestiones de teoría y método. Suplementos Anthropos 45. Anthropos. Barcelona, España.

Ídem [coord] (1995), Determinismos y alternativas en las ciencias sociales de América Latina. CRIM-UNAM/Nueva Sociedad. Caracas, Venezuela.

Ídem (1996), Problemas antropológicos y utópicos del conocimiento. El colegio de México. México D.F, 1996.

Ídem (1998), Sujeto: existencia y potencia. Anthropos. Barcelona, España.

Ídem (2002), Necesidad de conciencia. Un modo de construir conocimiento. Anthropos. Barcelona, España.

Ídem (2005), Voluntad de conocer. El sujeto y su pensamiento en el paradigma crítico. Anthropos. Barcelona, España.

Ídem (2007), El ángel de la historia: determinación y autonomía de la condición humana. Anthropos. Barcelona, España.

Ídem (2011a), Configuraciones Críticas. Pensar epistémico sobre la realidad. Siglo XXI/Crefal. México D.F, México.

Ídem (2011b), Horizontes de la razón III: el orden del movimiento. Anthropos. Barcelona, España.

Zemelman, Hugo y León, Emma [coord] (1997), Subjetividad: umbrales del pensamiento social. Anthropos. Barcelona, España.

Zemelman, Hugo y Quintar, Estela (2007), Conversaciones acerca de interculturalidad y conocimiento. Ipecal/Instituto Politécnico Nacional. México D. F, México.

Recibido: 16.11.2013

Aprobado: 13.12.2013 